\title{
Predictability of Rules in HIV-1 Protease Cleavage Site Analysis
}

\author{
Hyeoncheol Kim ${ }^{1}$, Tae-Sun Yoon ${ }^{1}$, Yiying Zhang ${ }^{2}$, \\ Anupam Dikshit ${ }^{2}$, and Su-Shing Chen ${ }^{2}$ \\ ${ }^{1}$ Dept. of Computer Science Education, Korea University, \\ Seoul, 136-701, Korea \\ hkim@comedu.korea.ac.kr \\ ${ }^{2}$ Computer \& Info. Sciences and Engineering, University of Florida, \\ Gainesville, FL. 32611, U.S.A. \\ suchen@cise.ufl.edu
}

\begin{abstract}
Symbolic rules play an important role in HIV-1 protease cleavage site prediction. Recently, some studies have done on extraction of the prediction rules with some success. In this paper, we demonstrated a decompositional approach for rule extraction from nonlinear neural networks. We also compared the prediction rules to the ones extracted by other approaches and methods. Empirical experiments are also shown.
\end{abstract}

\section{Introduction}

HIV-1 protease is an enzyme in the AIDS virus that is essential to its replication. Understanding HIV-1 protease cleavage site specificity is very desirable, because efficiently cleaved subtrates are also excellent templates for synthesis of tightly binding chemically modified inhibitors. HIV-1 protease inhibitor drugs are small molecules that bind to the active site in HIV-1 protease and stay there, so that the normal functioning of the enzyme is prevented.

Recently, many machine learning methods have been applied to the problem of HIV-1 protease cleavage site prediction [2, 3, 8, 9, 12, 7. Neural networks have been successful for the cleavage site prediction yielding a prediction accuracy of $90-92 \%$, which is superior than decision tree C5.0 of $85.5 \%$ and similar to Gaussian SVM 2, 8, 3. Linear classifiers such as a perceptron and linear SVM were also used yielding accuracy of $88-92 \%$ while MLP produced accuracy of 86-92\% according to size of training dataset [9].

Narayanan et al. addressed importance of symbolic interpretation in HIV-1 protease cleavage site prediction, and they demonstrated symbolic rule generation using decision trees 8 . Rules can guide future in vitro experiments which take into account various patterns of residues and identify the most important positions to mutate in the n-residue regions to test for cleavability [8, 9, Even though their experiments showed that neural networks outperformed decision trees, they used a decision tree to generate symbolic rules because MLP is hard to interprete. Rögnvaldsson et al. used a linear model such as a simple perceptron or LSVM to generate rules because it is more easily interpreted than a nonlinear 
one such as MLP. Rögnvaldsson et al. used a so-called exhaustive search method to generate rules from simple linear perceptron, in which they generated all possible candidate combinations and tested each one with a trained perceptron that is used as a function. However, this method is not practical for nonlinear model (i.e., MLP).

In this paper, we applied a decompositional approach to extract symbolic rules from trained nonlinear neural networks (i.e., MLP), which is computationally practical and safe. Also we compared the extracted rules with others from various methods in terms of their predictability.

\section{$2 \quad$ Rule Discovery Using Neural Networks}

As addressed in [8, machine learning algorithm needs to take into account combined influence of attributes on classification. Algorithms such as MLP do so by adjusting all the connection weights for each instance to ensure more accurate classification, while decision tree looks for just one attribute (i.e., the most important residue position) at each decision point in the tree on which to split the samples. However the knowledge learned by MLP can not be converted easily into symbolic rules.

In neural networks, since the knowledge learned through training is distributed to connection weights that are interconnected nonlinearly, it is very hard to interpret the black box into human-understandable symbolic forms. In recent years, there have been studies on rule extraction from feed-forward neural networks [1, 4, 5, 6, 10, 11]. The extracted rules provide human users with the capability to explain how the patterns are classified and may provide better insights about the domain.

There are two approaches of rule extraction from neural networks(i.e., MLP): pedagogical (black-box) approach and decompositional (white-box) approach. Pedagogical approaches take a trained neural network as a black-box and observe input patterns and their corresponding output patterns. Generic methods of the approaches take into account all possible combinations of an input vector which are then presented to the trained neural network for their validity evaluation. For our cleavage site prediction problem, domain size would be $20^{8}$ and the number of all possible candidate rules would be $21^{8}$. Also there are some complicated efficiency issues on how to evaluate a candidate rule and how to generate maximally general and accurate rules. Obviously, pedagogical approaches are not effective when the size of the neural network increases. In order to overcome this limitation, decompositional approaches apply heuristically guided search to the process of rule extraction.

Decompositional approaches to rule extraction from a trained neural network (i.e., MLP) involves the following phases:

1. Intermediate rules are extracted at the level of individual units within the network (i.e., hidden and output nodes).

2. The intermediate rules from each unit are aggregated to explain the logical mapping between the input and output nodes of the network. 


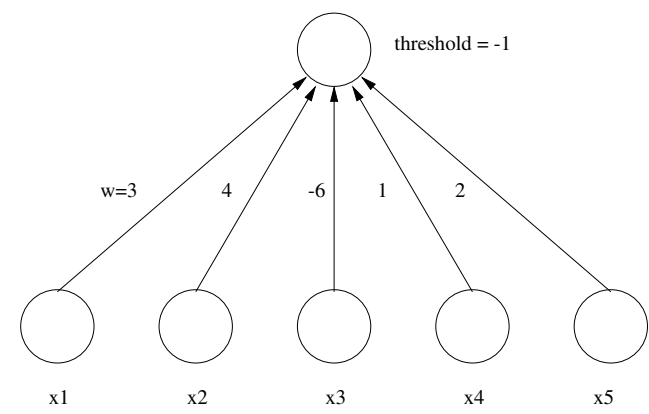

Fig. 1. An individual rule with five incoming connection weights and a threshold

At the first phase, it searches valid and maximally general (i.e., size of a combination is as small as possible) combinations by finding the combinations of weights of a node that will make it active. It is illustrated in figure 1 in which a non-input unit with 5 binary inputs, their corresponding incoming connection weights and a threshold is given. For example, a combination $\left(x_{1}, x_{2}\right)$ is a valid rule because its weight-sum (i.e., $3+4=7$ ) is always greater than the threshold (i.e., -1 ) regardless of the values of other incoming units.

However the process in phase 1 is inherently combinatorial. For $n$ binary inputs, the number of combinations is $3^{n}$ (i.e., exponential complexity) which is computationally very expensive as the $n$ grows. Thus, heuristics have been proposed to reduce the search space. We used Kim's OAS algorithm for our experiments, which orders incoming weights by their contributions to node activity and then searches valid and maximally-general rules using tree-based search structure in $O(n \log n)$ most cases and less than $O\left(2^{n-1}\right)$ worst case [6]. Our implementation of the OAS algorithm uses two parameters to control the quantity and quality of the rules extracted: $k$ and $C F$ threshold. At each node, it generates $k$ rules with the highest $\mathrm{CF}$ values among all the rules whose $\mathrm{CF}$ values are greater than the $C F$ threshold. The $\mathrm{CF}$ value is defined as the output value of sigmoid function in the node. CF threshold is normally set to 0.8 for positive rules.

Decompositional approaches take each node in the network as a concept unit and the concepts in the concept node are logically aggregated with other concepts from other nodes. Thus, the approaches using the parameters can generate general rules excluding many other trivial rules. Therefore the rules extracted by this approach can be different from the rules by black-box approaches.

\section{Experimental Results}

\subsection{Datasets}

Cai and Chou 2] published a dataset with 362 peptides in 1998, which has been used as the standard dataset in many publications of this research domain [8, 9, 12. The data set includes 362 examples with 114 positives (i.e., cleaved) and 
Table 1. HIV-1 protease cleavage site datasets. Default cleavability represents probability of the cleaved of a default rule (i.e., "Whatever it is, it is always cleaved").

\begin{tabular}{l|cc|cc}
\hline Dataset & Cleaved & Uncleaved & Total & $\begin{array}{c}\text { Default } \\
\text { cleavability }\end{array}$ \\
\hline Cai et al. & 114 & 248 & 362 & $31.49 \%$ \\
New dataset & 281 & 111 & 392 & $71.68 \%$ \\
\hline Combined & 395 & 359 & 754 & $52.52 \%$ \\
\hline
\end{tabular}

248 negatives (i.e., non-cleaved). Recently, You et al. collected 384 more peptides to create a 746-peptide dataset [12. Since the new dataset is not available to public domain currently, we created our own new dataset in a similar way by collecting and refining peptide sequences from published literatures containing experimental data on oligopeptide sequences that have been exposed to the HIV1 protease 12. We collected 392 new examples and thus created a 754-peptide dataset without duplication. In this article, we call the datasets as a 362-dataset, a 392-dataset and a 754-dataset, respectively. Table 1 shows their distributions.

A sequence in the datasets is composed of 8 positions and each position represents one of 20 different amino acids. A peptide is denoted by $p_{4} p_{3} p_{2} p_{1} p_{1}^{\prime} p_{2}^{\prime} p_{3}^{\prime} p_{4}^{\prime}$ where each $p$ represents one of 20 amino acids. The scissile bond is located between positions $p_{1}$ and $p_{1}^{\prime}$. For our experiments, we use 9 attributes for each octapeptide: location 1 though location 8 and class attribute (i.e., cleavagability). Location 1 corresponds to $p_{4}$ and location 8 to $p_{4}^{\prime}$. We also used orthonormal encoding scheme that maps the sequence to a sparse orthonormal representation. Each amino acid $p_{i}$ is then represented by a 20 bit vector with only one bit set to one and the rest 19 bits to zero. This places an octamer in one of the corners of a $20 * 8=160$ dimensional hypercube.

Like other cleavage site prediction domains, the HIV-1 protease cleavage domain has a problem of dataset size. That is, the number of available sequences is too small compared to domain size: $362 \ll 2^{8} \ll 2^{160}$. And there is no evidence that the 362 or 754 examples are representative for the domain. Thus, a question comes to us that classifiers created from just 362 or 754 examples can really classify any previously unknown instance that is picked from the space of $20^{8}$ instances.

\subsection{Rule Extraction and Comparison}

In our experiments, we first extract rules using different approaches and methods, and then compare quality of the rules with their predictability. The predictability of a classifier means how well a classifier created by a small number of examples can predict other instances previously unknown.

We used the 362-dataset as a training set, and the 392-dataset as a test set. Since the rules published in other papers have been created on the 362-dataset 8, 9, 7, we used the same training dataset to generate our rules and used the new 392-dataset for testing all the rules together. 
Table 2. The rules extracted by See5 [8]. Positions @1-4 represent the left hand sequence in front of cleavage point and positions @5-8 the right hand sequence. TP for true positives and FP for false positives. Rule 1, 2, 3 are generated by normal mode and rule 4 is generated by boosting mode.

\begin{tabular}{l|l|c|c|c}
\hline & $\begin{array}{l}\text { DT Rules } \\
\text { on 362-dataset }\end{array}$ & $\begin{array}{c}\mathrm{P} / \mathrm{FP} \\
\text { on 362-dataset } \\
(362 / 248)\end{array}$ & $\begin{array}{c}\mathrm{P} / \mathrm{FP} \\
\text { on 392-dataset } \\
(392 / 111)\end{array}$ & $\begin{array}{c}\mathrm{P} / \mathrm{FP} \\
\text { on combined set } \\
(754 / 359)\end{array}$ \\
\hline 1 & F@4, cleavage & $35 / 5$ & $42 / 4$ & $77 / 9$ \\
2 & L@4, cleavage & $38 / 9$ & $47 / 4$ & $85 / 13$ \\
3 & Y@4 and P@5, cleavage & $32 / 5$ & $44 / 14$ & $76 / 19$ \\
4 & E@6, cleavage & $38 / 6$ & $17 / 0$ & $55 / 6$ \\
\hline
\end{tabular}

Narayanan, et al. 8] tried a decision tree C5.0 to extract prediction rules for HIV cleavage site, which are listed in table 2 Position 4 and 5 are considered to be important attributes by the decision tree. A decision tree searches the most informative attribute first and divides examples by the attributes, and then do the same with the subgroup of the examples. The most informative attribute is determined by information gains of attributes, and the information gain is obtained by amino acid's frequency in the examples. Thus, the extracted rules are attribute (i.e., position)-oriented and frequency-based. Frequency-based (or probabilitybased) mining normally requires a training set to be large enough and/or training examples to be high domain-representative. With the 362-dataset whose quantity and quality of examples are not good enough, quality of the rules extracted directly from the examples might not be guaranteed.

We also tried association rule mining which is another frequency(or probability)-based rule mining method. While decision trees search for attributes (i.e., positions) one by one to generate rules, association rule mining searches for a set of attributes that are associated each other. The association is defined by frequency of co-occurrence of the attributes in a set. The frequency-based association rules are shown in table 3. The frequency-based method is statistically meaningful when the size of data samples are large enough. Since the size HIV data set is not large enough, the generated rules does not cover all of meaningful patterns in the domain as seen in table 3

A feed-forward neural network trained by backpropagation is not a frequentbased attribute-by-attribute method. A perceptron uses gradient descent-based learning for linear classification, and multi-layered perceptron is a set of perceptrons that are networked in a layered architecture and thus it provides nonlinear separability by combining linear separable perceptrons. Rögnvaldsson et al.

Table 3. Association rules

\begin{tabular}{c|c|c|c|c}
\hline & Assoc. Rules on a 362-dataset & support count & confidence & correlation \\
\hline 1 & P@5, cleavage & 60 & $80.0 \%(60 / 12)$ & 2.54 \\
2 & E@6, cleavage & 38 & $84.2 \%(38 / 6)$ & 2.67 \\
\hline
\end{tabular}


Table 4. Rules extracted from a simple linear perceptron trained on a 362-dataset 9 . 6 other rules containing E@6 are subsumed to the rule1.

\begin{tabular}{|c|c|c|c|c|}
\hline & $\begin{array}{l}\text { SP Rules } \\
\text { on 362-dataset }\end{array}$ & $\begin{array}{c}\mathrm{P} / \mathrm{FP} \\
\text { on 362-dataset }\end{array}$ & $\begin{array}{c}\mathrm{P} / \mathrm{FP} \\
\text { on 392-dataset }\end{array}$ & $\begin{array}{c}\mathrm{P} / \mathrm{FP} \\
\text { on combined set }\end{array}$ \\
\hline 1 & E@6, cleavage & $38 / 6$ & $17 / 0$ & $55 / 6$ \\
\hline 2 & F@4 and P@5, cleavage & $17 / 0$ & $3 / 0$ & $20 / 0$ \\
\hline 3 & F@4 and Q@6, cleavage & $14 / 0$ & $8 / 0$ & $22 / 0$ \\
\hline 4 & F@4 and V@3, cleavage & $0 / 0$ & $20 / 1$ & $20 / 1$ \\
\hline 5 & L@4 and Q@6, cleavage & $3 / 0$ & $1 / 0$ & $4 / 0$ \\
\hline
\end{tabular}

used a exhaustive rule search method which is a generic black box approach to generate rules from a trained simple perceptron which is a linear model [9, 12. In an exhaustive rule search, all possible combinations of an input vector are taken into account in the process of rule generation. For each combination (i.e., candidate rule), it is presented to the trained neural network to get its validity. Even for 2-position rules only (i.e., size of antecedent of a rule is 2), there are ${ }^{8} C_{2} * 20 * 20=11,200$ candidate rules to test. They generated all the cases and ranked them by their probabilities obtained from a trained linear perceptron. Table 4 lists the 2-position rules. They showed top 10 rules [9], but we generalized 6 rules containing "E@6" in their antecedents into a 1-position rule (i.e., rule1 in table 4). Since the rules in table 4 are top-10 rules with the highest probabilities among 11,200 combinations, rules with very high accuracy are first selected.

Table 5. The rules extracted by a neural network trained on a 362-dataset. CF threshold at each hidden unit was set to 0.6 for the top table and 0.8 for the bottom table. The lower the CF threshold is, the more general rules are generated.

With CF threshold $=0.6$

\begin{tabular}{c|l|c|c|c}
\hline & NN Rules & P/FP & P/FP & P/FP \\
& on a 362-dataset & on 362-dataset & on 392-dataset & on combined set \\
\hline 1 & P@5, cleavage & $60 / 12$ & $66 / 28$ & $126 / 40$ \\
2 & L@4, cleavage & $38 / 9$ & $47 / 4$ & $85 / 13$ \\
3 & E@6, cleavage & $38 / 6$ & $17 / 0$ & $55 / 6$ \\
4 & N@3, cleavage & $78 / 31$ & $96 / 30$ & $174 / 61$ \\
5 & V@3, cleavage & $33 / 13$ & $138 / 13$ & $171 / 26$ \\
6 & F@4, cleavage & $35 / 5$ & $42 / 4$ & $77 / 9$ \\
7 & A@6, cleavage & $28 / 22$ & $83 / 3$ & $111 / 25$ \\
\hline
\end{tabular}

\begin{tabular}{|c|c|c|c|c|}
\hline \multicolumn{5}{|c|}{ With CF threshold $=0.8$} \\
\hline & $\begin{array}{l}\text { NN Rules } \\
\text { on a 362-dataset }\end{array}$ & $\begin{array}{c}\mathrm{P} / \mathrm{FP} \\
\text { on 362-dataset }\end{array}$ & $\begin{array}{c}\mathrm{P} / \mathrm{FP} \\
\text { on 392-dataset }\end{array}$ & $\begin{array}{c}\mathrm{P} / \mathrm{FP} \\
\text { on combined set }\end{array}$ \\
\hline 1 & E@6, cleavage & $38 / 6$ & $17 / 0$ & $55 / 6$ \\
\hline 2 & F@4 and $\mathrm{P} @ 5$, cleavage & $17 / 0$ & $3 / 0$ & $20 / 0$ \\
\hline 3 & N@3 and F@4, cleavage & $17 / 1$ & $13 / 1$ & $30 / 2$ \\
\hline 4 & $\mathrm{~N} @ 3$ and $\mathrm{A} @ 1$, cleavage & $8 / 1$ & $1 / 1$ & $9 / 1$ \\
\hline 5 & $\mathrm{~N} @ 3$ and $\mathrm{A} @ 6$, cleavage & $2 / 1$ & $2 / 0$ & $4 / 1$ \\
\hline 6 & V@3 and A@6, cleavage & $4 / 2$ & $56 / 1$ & $60 / 3$ \\
\hline
\end{tabular}


However, the exhaustive method is not practical at all in terms of its computational complexity, and it is also very difficult to generate rules from non-linear classifiers such as MLP. Thus, it is not practical to use a black-box approach to generate rules from a non-linear model such as MLP.

We used a decompositional approach to extract rules from MLP neural networks with 8 hidden units. The extracted rules from the MLP with two different $C F$ threshold parameter values are listed in table 5 . We could extract more general rules from neural networks. Notice that the rules " $A @ 6$ " and "V@3 and $A @ 6$ " show low accuracy on training set but very high prediction accuracy on test set. Frequency-based methods such as decision trees can not generate this kind of rules because the methods are more dependent on training examples. The rule"E@6" was discovered by any methods, and the rule "F@4 and P@5" which is already well known by domain experts was discovered by neural network methods.

\section{Conclusion and Discussion}

Narayanan et al. [8] used a decision tree to generate symbolic rules for HIV-1 protease cleavage site prediction because they could not interpret neural network knowledge in symbolic format even though neural networks outperform decision trees. Because of the small number of examples available, probability (or frequency)-based mining (i.e., decision trees and association rule mining) might be misleading if the examples are not high domain-representative. Rögnvaldsson et al. [9, 12] used linear perceptron to generate symbolic rules because nonlinear multilayered perceptron (i.e., MLP) is hard to be interpreted. They had to show that linear model performs as good as the nonlinear model before they used the linear model to generate rules. However, the exhaustive method they used is not computationally practical and have some efficiency issues on rule generation. Also we don't have any clear evidence that the HIV-1 protease cleavage site prediction problem is naturally linear problem.

In this paper, we applied a decompositional approach to extract symbolic rules from a trained nonlinear neural network(i.e., MLP), and compared the rules to the ones extracted by other methods. Experimental results show that a neural network works better especially when only small number of training examples are available. The extracted rules from the MLP seems to have good generalization capability and thus predictability.

\section{References}

1. Robert Andrews, Joachim Diederich, and Alan B. Tickle. Survey and critique of techniques for extracting rules from trained artificial neural networks. KnowledgeBased Systems, 8(6):373-389, 1995.

2. Yu-Dong Cai and Kuo-Chen Chou. Artificial neural network model for predicting HIV protease cleavage sites in protein. Advances in Engineering Software, 29:119$128,1998$. 
3. Yu-Dong Cai, X.J. Liu, X.B. Xu, and Kuo-Chen Chou. Support vector machines for predicting hiv protease cleavage sites in protein. journal of Computational Chemistry, 23:267-274, 2002.

4. LiMin Fu. Neural Networks in Computer Intelligence. McGraw Hill, Inc., 1994.

5. LiMin Fu. Rule generation from neural networks. IEEE Transactions on Systems, Man, and Cybernetics, 24(8):1114-1124, 1994.

6. Hyeoncheol Kim. Computationally efficient heuristics for if-then rule extraction from feed-forward neural networks. Lecture Notes in Artificial Intelligence, 1967:170-182, 2000.

7. Alessandra Lumini and Nanni Loris. Machine learning for hiv-1 protease cleavage site prediction. Proceedings of Artificial Intelligence and Application (AIA2005), 2005.

8. Ajit Narayanan, Xikun $\mathrm{Wu}$, and Z. Rong Yang. Mining viral protease data to extract cleavage knowledge. Bioinformatics, 18(Suppl.1):S5-S13, 2002.

9. Thorstein Rögnvaldsson and Liwen You. Why neural networks should not be used for HIV-1 protease cleavage site prediction. Bioinformatics, 20(11):1702-1709, July 2004 .

10. Rudy Setino and Huan Liu. Understanding neural networks via rule extraction. Proceedings of the 14th International Conference on Neural Networks, (1):480-485, 1995.

11. Ismali A. Taha and Joydeep Ghosh. Symbolic interpretation of artificial neural networks. IEEE Transactions on Knowledge and Data Engineering, 11(3):443-463, 1999.

12. Liwen You, Daniel Garwicz, and Thorstein Rögnvaldsson. Comprehensive bioinformatic analysis of the specificity of human immunodeficiency virus type 1 protease. Journal of Virology, 79(19):12477-12486, Oct 2005. 